\title{
Centers of Research Excellence
}

National Cancer Institute

\section{Source}

National Cancer Institute. Centers of Research Excellence. NCI Thesaurus. Code C19983.

Multidisciplinary and translational research teams focused on a specific disease, modality, biologic process or scientific area of particular significance. They are awarded sizeable amounts of flexible funding to enable them to rapidly address emerging scientific opportunities or specific challenges. 